\title{
Die Biomechanik der Wirbelsäule als Diktum für das Training des Pferdes
}

\author{
Das Pferd kommt nicht als Reitpferd auf die Welt
}

Katja Eser

\section{๑) Zusammenfassung}

Bei Rittigkeitsproblemen, Lahmheit, Verhaltensauffälligkeiten und Organfunktionsstörungen ist neben einer schulmedizinischen Abklärung auch eine osteopathische und energetische Befundung angezeigt, um das Pferd auch ursächlich in seine körperliche, energetische und psychische Balance zu bringen. Erfahrungsgemäß entwickeln sich die meisten Beschwerden im Bewegungsapparat auf der Grundlage eines unphysiologischen Trainings, das bei Fortsetzung dazu führt, dass das Pferd immer wieder die gleichen oder ständig wechselnde Symptome entwickelt, die langfristig dazu führen können, dass es früher als von der Natur vorgesehen sein Leben beenden muss. Zur langfristigen Wiederherstellung der Lebens- und Bewegungsfreude sollte die eigentliche tiefgreifende manuelle Behandlung stets von einer biomechanisch-physiologischen Gymnastizierung begleitet werden, zunächst durch den Therapeuten, nach entsprechender Einweisung dauerhaft dann durch den Reiter selbst.

\section{Die natürliche Balance des Pferdes}

Das Pferd hat sich im Laufe seiner Evolution von einem etwa $50 \mathrm{~cm}$ großen 5-Zehengänger zu einem bis zu $193 \mathrm{~cm}$ (Clydesdale) großen Mittelzehengänger entwickelt, dessen Hauptmerkmale seines Bewegungs- und Sozialsystems die Flucht und die Herde sind.

Alle Gelenke und Muskeln des Pferdes sind so aufgebaut und angelegt, dass es mit möglichst wenig Energieverbrauch lange stehen, im Schritt zügig und ausdauernd wandern und bei Bedarf eine explosionsartige Flucht nach vorne oder zur Seite antreten kann. In der Natur bewegt es sich aus Gründen der Energieeffizienz fast ausschließlich im Schritt. Trab und Galopp kommen relativ selten vor, dann v.a. in Situationen der Flucht, des Spiels und des Imponierens.

Von seiner Anatomie und Physiologie her betrachtet ist das Pferd ein Lauftier. In der Fortbewegung drückt jeweils ein Hinterbein in den Boden und schiebt den Körper nach vorne. Dadurch verschiebt sich auch der Schwerpunkt des Pferdes nach vorne. Um zu verhindern, dass es ,auf die Nase fällt", sucht sich ein Vorderbein eine neue kraniale Unterstützungsfläche. Im natürlichen Gang erfolgen somit die Schritte der Vorhand passiv als Folge des aktiven Vortretens der Hinterhand. Die Wirbelsäule hat als Bindeglied zwischen Hinter- und Vorhand die Funktion, den Schub der Hinterhand aktiv nach kranial fortzuleiten. Im Gegensatz zur Rückenmuskulatur des Menschen, die eine statische Haltefunktion gegen die Schwerkraft hat, ist die Rückenmuskulatur des Pferdes eine dynamisch arbeitende Bewegungsmuskulatur. Somit ist der Rücken des Pferdes primär nicht dafür gebaut, einen Reiter zu tragen.

Der physiologische Schwerpunkt des Pferdes liegt etwa eine Handbreit kaudal des Olekranons auf Höhe des Buggelenks. Hierdurch befinden sich ca. 65\% des Körpergewichts auf der Vorhand und etwa 35\% auf der Hinterhand. In dieser für das Tier natürlichen Situation befindet es sich in Balance mit sich selbst.

\section{Der Reiter als Störfaktor der Balance}

In dem Moment, in dem sich ein Mensch auf den Rücken eines Pferdes setzt, kommt das Pferd aus seiner natürlichen Balance. Wir Menschen können dies selbst gut nachvollziehen, indem wir uns ein Kind auf die
Schultern setzen: Unser neu entstandener gemeinsamer Schwerpunkt liegt deutlich kranialer als unser eigener Schwerpunkt. Durch die neu entstandene längere „Körpergröße“ haben wir einen viel größeren Hebel-/Lastarm in der Standbeinphase des Gehens auszubalancieren.

Folgende Strategie des Pferdes, mit dem Reitergewicht zurechtzukommen, ist weit verbreitet: Beim Aufsitzen des Reiters behält das Pferd lediglich seine normale Grundkörperspannung bei, die das zusätzliche Reitergewicht allerdings nicht kompensiert. Somit hängt der Rücken des Pferdes nach ventral durch. Dies führt nach einiger Zeit zu Schmerzen aufgrund der Überdehnung und Ermüdung der ventralen Rückenmuskel- und Bauchmuskelanteile. Um diese zu entlasten, fällt das Pferd auseinander, d.h. die Vorhand wird weit nach vorne, die Hinterhand weit nach hinten ausgestellt. Dadurch kommen die sehnigen ventralen Bandstrukturen zwischen Vorhand-Rumpf-Hinterhand auf Zug und helfen mit, das Reitergewicht weiterhin ohne nennenswerten Muskeleinsatz zu tragen. Reicht dies nicht aus, spannt das Pferd die gesamte Rücken- und Bauchmuskulatur in dieser Überdehnung an und versteift damit seinen Rumpf. Der Rücken wird fest, die Tritte werden kurz, klemmig und schwunglos. Die nach einer gewissen Zeit folgende Ermüdung der Rücken- und Bauchmuskeln versucht das Pferd zu beenden, indem es wiederum seinen Rücken durchhängen lässt. Im schlimmsten Fall kann ein Pferd sein gesamtes Reitpferdeleben lang zwischen Rücken-Verspannen und Rücken-Wegdrücken verbringen.

Die möglichen Folgen dieser mangelhaften Ausbildung der Muskulatur und der gesunderhaltenden Bewegungsabläufe eines Reitpferds sind häufig: 
- segmentale Verspannungen und Blockierungen von Genick, Wirbelsäule und Iliosakralgelenken

- Sehnenschäden der Vorderbeine aufgrund des unphysiologisch hohen Gewichts auf der Vorhand

- Gelenksschäden der Hinterbeine aufgrund der unphysiologischen Winkelung der ausgestellten Hinterhand

\section{Die Wirbelsäule des Pferdes als Bogensehnenbrücke}

Die Wirbelsäule des Pferdes ist aufgebaut wie eine Art Bogensehnenbrücke, die sich zusammensetzt aus

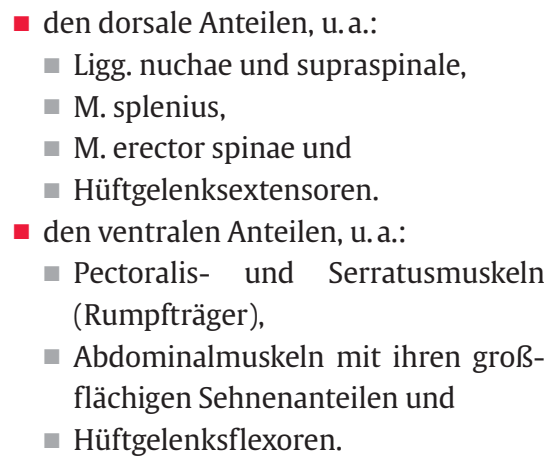

Diese Bogensehnenbrücke trägt sich selbst, wenn von kranial und kaudal Zug in Form von Dehnung auf die sehnigen Anteile der dorsalen Verspannung ausgeübt wird. Das Erarbeiten der vertikalen Balance (s. unten) führt zu einem Aufwölben des Rückens mit Dehnung des Nacken-Rückenbands (Ligg. nuchae und supraspinale), sodass sich der Pferderumpf in seinen sehnigen Anteilen „aufhängen“ kann. Im Gegensatz zum „Durchhängen“ des Rückens beim unphysiologisch trainierten Pferd werden hier die ventralen Strukturen nicht überdehnt, sondern aktiviert. Dies ermöglicht eine Entspannung der langen und großen Rückenmuskeln, sodass diese ihrer physiologischen Aufgabe nachkommen und den Schub der Hinterhand durch die schwingenden Wirbelsegmente aktiv nach kranial fortleiten können.

\section{Wiederherstellung der Balance des Pferdes}

Um das Pferd mit (!) dem Reiter wieder in Balance zu bringen, ist es erforderlich, das Pferd zu befähigen, in der Hinterhand die vermehrte Last aufzunehmen, um die der Reiter die Vorhand beschwert. Das heißt:
Die Hinterbeine müssen mehr belastet werden, um über eine schwingende Wirbelsäule die Vorhand zu entlasten (= relative Aufrichtung). Das Reiten eines Pferdes stellt also in gewisser Weise eine paradoxe Situation dar: Man muss ein Pferd korrekt reiten, um bei ihm die Muskeln aufzubauen, die es nur dafür braucht, um einen Reiter tragen zu können und dabei gesund zu bleiben. Hier zeigt sich der große Irrtum mancher Reiter in der Annahme, sie würden ihren Pferden etwas Gutes tun, wenn sie am durchhängenden Zügel ohne Aktivierung des Nacken-Rückenbandes „durchs Gelände bummeln“.

Das Ausbilden der Balance und der Geschmeidigkeit erfolgt über die physiologische Gymnastizierung des Pferdes an der Hand und unter dem Sattel in horizontaler und vertikaler Richtung entsprechend der Biomechanik der Wirbelsäule und der Extremitätengelenke. Zentrale Punkte dieses Trainings sind:

- Bewegung und Belastung der Wirbelsegmente und Extremitätengelenke entsprechend ihrer gelenkspezifischen Kraftwirkungslinien und Gelenkachsen

- Förderung einer gesunden Muskelfunktion, die es einem Muskel je nach Erfordernis ermöglicht, sich jederzeit anzuspannen, zu entspannen und zu dehnen

- Förderung der Tragkraft der Hinterhand zur Entwicklung der relativen Aufrichtung

a Förderung der Bewegungs- und Lebensfreude des Pferdes mit einer stabilen und gelassenen Psyche

\section{Vertikale Balance und ihre Biomechanik}

Vertikale Balance bedeutet das Finden des Schwerpunkts zwischen kranial-kaudal (direkt) und damit indirekt auch zwischen dorsal-ventral (reelle Dehnungshaltung und relative Aufrichtung). Die vertikale Balance entwickelt sich über die Dehnungshaltung nach vorwärts-abwärts mit Fallenlassen des Halses aus einem zwischen den Schulterblättern angehobenen Widerrist und gleichzeitiger Hankenbeugung.

Essenziell für die vertikale Balance ist die koordinierte Tätigkeit der ventralen und dorsalen Muskelketten miteinander. Agonisten, Synergisten und Antagonisten arbeiten der jeweiligen Haltung und Bewegung entsprechend mit dem jeweils erforderlichen Maß an Spannung, Entspannung und Dehnung.

Die kraniale Komponente der Dehnungshaltung ergibt sich aus der S-förmigen Schwingung der Halswirbelsäule (HWS) mit ihrem Umkehrpunkt auf Höhe C3/C4. Kranial dieses Umkehrpunkts schwingt die HWS kyphotisch, kaudal dieses Umkehrpunkts schwingt sie lordotisch und geht mit ihrem Scheitelpunkt C7 in die Brustwirbelsäule (BWS) über, die kaudal des Widerrists in die Kyphose „umschwingt“.

In der korrekten Dehnungshaltung nach vorwärts-abwärts entfaltet sich dieser S-förmige Schwung, der kyphotische Anteil streckt sich, die Ganasche öffnet sich, der lordotische Anteil beugt sich, die nach kaudal zeigenden Dornfortsätze richten sich aufgrund der Spannung von Ligg. nuchae und supraspinale auf. Bei einem derartigen korrekten Fallenlassen des Halses aus einem angehobenen Widerrist heraus wird die Vorhand „leichter“.

Fehlerhafte, da nicht zur Dehnung des Lig. nuchae führende „Dehnungshaltungen“ entstehen unter anderem:

- Bei der Hyperflexion der HWS: Hier wird zwar der lordotische Anteil der HWS gebeugt, aber der kyphotische Anteil noch weiter gebeugt statt gestreckt, sodass es zu keiner Entfaltung der S-Form kommt und die Ganasche sich noch weiter schließt (häufig gesehen im Dressurreitsport).

- Beim Senken des Halses bei gleichzeitigem Absenken des Widerrists zwischen den Schulterblättern: Durch das Absenken des Widerrists kommt das Lig. nuchae nicht auf Dehnung, sondern im Gegenteil in Annäherung, die Vorhand wird „schwerer“ (häufig gesehen im Westernreitsport).

Die kaudale Komponente der Dehnungshaltung ergibt sich aus der Hankenbeugung, d.h. der Flexion im Becken, den Iliosakral-(ISG-) und Hüftgelenken. Das Lig. supraspinale kommt auf Dehnung und die nach kranial zeigenden Dornfortsätze richten sich auf. Die korrekte Hankenbeugung führt zu einer vermehrten Lastaufnahme der Hinterhand. Außerdem wird das Pferd von kaudal her kürzer, runder, tiefer, was im Verhältnis dazu die Vorhand 


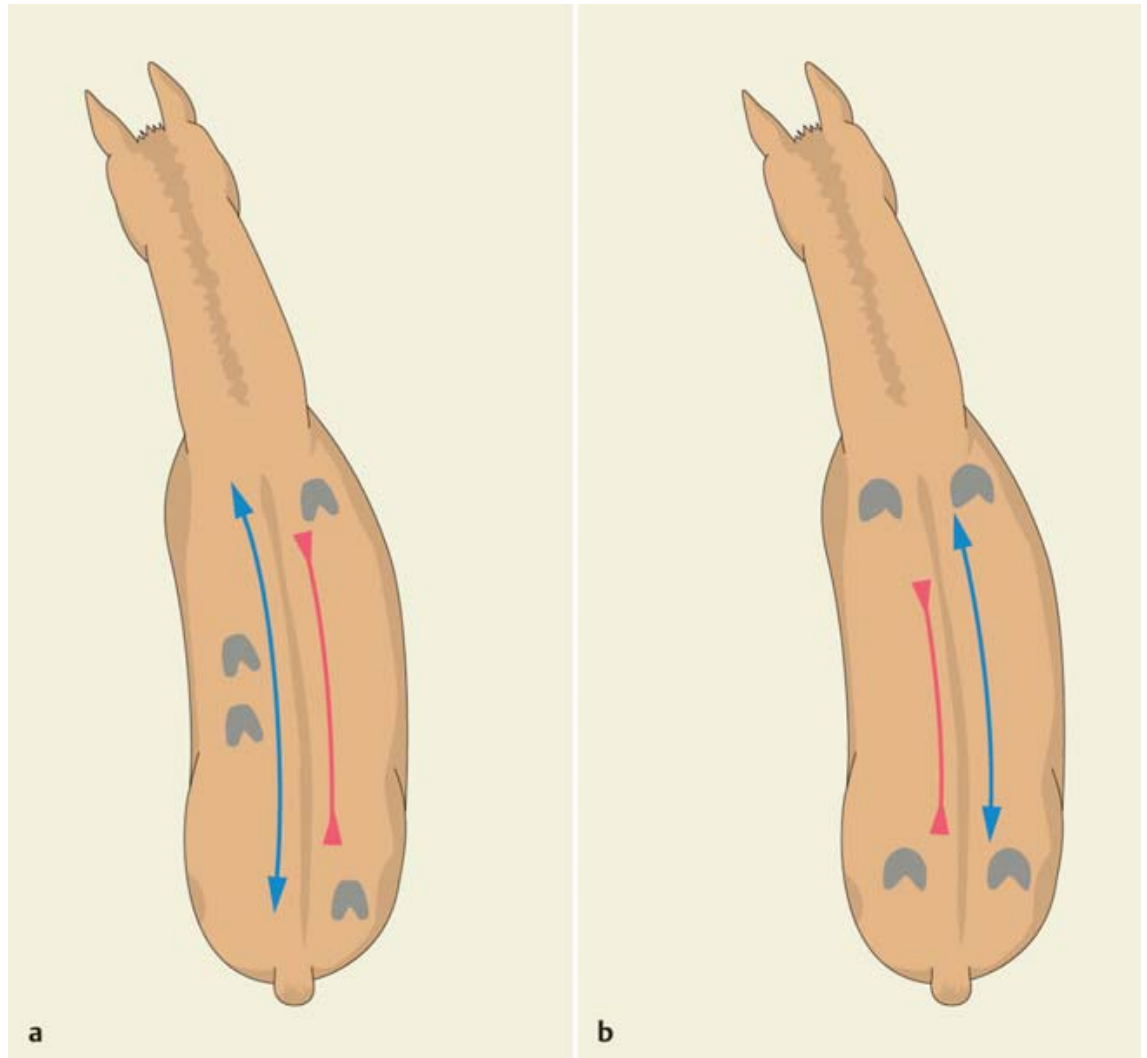

Abb. 1 a und $\mathbf{b}$ Wirbelsäule von dorsal (blau = gedehnt; rot = aktiv): a in der Fortbewegung, $\mathbf{b}$ im Stand. (c) Eser K. Checkliste Osteopathie Pferd. Stuttgart: Sonntag Verlag; 2011.

anhebt und entlastet (= relative Aufrichtung). Die Hankenbeugung kann u.a. durch das Beüben des Untertretens der Hinterhand unter den Schwerpunkt gefördert werden, sodass sich hier die vertikale und horizontale Balance treffen.

\section{Horizontale Balance und ihre Biomechanik}

Horizontale Balance bedeutet das Auffinden des Schwerpunkts zwischen lateralmedial-lateral (direkt) und damit indirekt auch zwischen dorsal-ventral (reelle Dehnungshaltung und relative Aufrichtung). Die horizontale Balance entwickelt sich über Stellung im Atlantookzipitalgelenk, Biegung im gesamten Körper mit Untertreten des inneren und/oder äußeren Hinterbeins unter den Schwerpunkt.

Essenziell für die horizontale Balance ist die koordinierte Tätigkeit der lateralen Muskelketten miteinander. Agonisten, Synergisten und Antagonisten arbeiten der jeweiligen Haltung und Bewegung entsprechend mit dem jeweils erforderlichen Maß an Spannung, Entspannung und Dehnung.

\section{Biomechanik von Rumpf und Becken in der Fortbewegung}

Die Wirbelsäule ist je nach Gangphase immer mindestens an einem Ende frei beweglich. Dazu ein Beispiel:

Das linke Hinterbein tritt vor = LATFLEX links (\$ Abb. 1 a)

- Tuber coxae des Spielbeins (links) fällt nach ventral und zieht nach kranial = Becken in LATFLEX links mit ROT rechts nuber die ISG wird die Beckenbewegung auf das Sakrum und von dort weiterlaufend in die gesamte Wirbelsäule nach kranial weitergegeben = Sakrum und Wirbelsäule in LATFLEX links mit ROT rechts

n die Procc. spinosi „fallen“ nach links $=$ Wirbel in ROT rechts

- der an der BWS aufgehängte Brustkorb nimmt die Wirbelsäulenbewegung auf = Brustkorb senkt sich links nach ventral und hebt sich rechts nach dorsal

- der Brustkorb schwingt nach rechts und gibt links den Raum frei für das nach vorne greifende linke Hinterbein

\section{Biomechanik von Rumpf und Becken im Stand}

Die Wirbelsäule ist an beiden Enden fixiert. Auch dazu ein Beispiel:

LATFLEX links im Stand ( Abb. 1 b)

- Tuber coxae links steigt nach dorsal und zieht nach kranial = Becken in LATFLEX links mit ROT links

über die ISG wird die Beckenbewegung auf das Sakrum und von dort weiterlaufend in die gesamte Wirbelsäule nach kranial weitergegeben = Sakrum und Wirbelsäule in LATFLEX links mit ROT links

- die Procc. spinosi „weichen“ nach rechts $=$ Wirbel in ROT links

- der an der BWS aufgehängte Brustkorb nimmt die Wirbelsäulenbewegung auf = Brustkorb hebt sich links nach dorsal und senkt sich rechts nach ventral - der Brustkorb schwingt nach links

\section{Biomechanik der HWS}

Initiierung von kaudal

in der Fortbewegung

In dieser Bewegung sind Kopf und HWS nur an der Halsbasis fixiert und an einem Ende frei beweglich. Die Biomechanik der HWS entspricht der Biomechanik von Rumpf und Becken von kaudal her in der 
Fortbewegung: LATFLEX und ROT gegensinnig setzen sich bis zum Okziput fort. Dadurch fällt der Mähnenkamm in die LATFLEX hinein.

\section{Passive Initiierung von kranial}

Dies ist zum Beispiel der Fall, wenn der Therapeut den Schädel des Pferdes in die Stellung führt. Dabei sind Kopf und HWS an beiden Enden fixiert. Die Biomechanik der HWS entspricht der Biomechanik von Rumpf und Becken im Stand mit LATFLEX und ROT gleichsinnig. Der Mähnenkamm stellt sich auf bzw. fällt aus der LATFLEX heraus.

Aktive Initiierung von kranial durch das Pferd selbst

Kopf und HWS sind nur an der Halsbasis fixiert und an einem Ende frei. Die Biomechanik der HWS entspricht der Biomechanik von Rumpf und Becken von kaudal her in der Fortbewegung mit LATFLEX und ROT gegensinnig. Der Mähnenkamm fällt in die LATFLEX hinein.

\section{Training der vertikalen und horizontalen Balance Theorie}

Um mit dem Pferd ein Training durchzuführen, das den gesamten Bewegungsund damit auch mittelbar den Organapparat gesund erhält oder gar rehabilitiert, sind folgende Aspekte zu beachten:

Bewegungsablauf entsprechend der Biomechanik

Die oben beschriebene Biomechanik von Genick, Wirbelsäule, Brustkorb und Becken im Stand und in der Fortbewegung sind das Maß der Bewegungsdurchführung. So sind während des Trainings stets die Rotationsrichtungen der Wirbelsegmente im Verhältnis zur Lateroflexionsrichtung im Auge zu behalten.

In diesem Zusammenhang stellt der Kopf des Pferdes ganz besondere Anforderungen an den Reiter bzw. Therapeuten, da der Kopf des Pferdes durch den Menschen viel leichter zu manipulieren ist als z.B. das Becken oder der Brustkorb. Eine Immobilisierung des Kopfes während der Fortbewegung (z. B. durch eine unnachgiebige Zügelführung) führt an der Halsbasis und/oder dem atlantookzipitalen Übergang zu einer Rotationsumkehr, die (mindestens) zu Läsionen der dort beteiligten Strukturen Atlas/Okziput/7. Halswirbel(C7)/1. Brustwirbel (Th1) und der Dura mater führen kann.

Darüber hinaus ist eine physiologische Haltung und Bewegung der Wirbelsäule essenzielle Basis für die mit ihr verbundenen Extremitäten und deren Bewegung und Belastung entsprechend ihrer physiologischen Gelenksachsen.

\section{Druck erzeugt Gegendruck}

Dynamik bedeutet: Wenn ein Agonist arbeitet, muss der Antagonist loslassen. Statik bzw. Isometrie bedeutet: Agonist und Antagonist arbeiten gleichzeitig.

Um ein gut vorstellbares Beispiel zu nutzen, soll hier die Bedeutung des ,lösenden Zügels“ im Gegensatz zum ,anstehenden Zügel“ verdeutlicht werden: Befindet sich ein Pferd in Stellung und Biegung, stellt sein Körper eine gebogene Linie dar, deren innere Seite verkürzt und deren äußere Seite verlängert ist. Von außen betrachtet ist nicht zu entscheiden, welche
Körperseite muskulär aktiv ist und welche muskulär loslässt. Dies lässt sich erst beurteilen, wenn man näher betrachtet, wie die Stellung und Biegung zustande kommen:

Der lösende Zügel gibt einen kleinen Impuls am Gebiss oder dem Kappzaum, der das Pferd dazu animiert, selbstständig aktiv die Stellung/Biegung einzunehmen und zu halten. Dies macht das Pferd, indem es auf der konkaven Seite die Muskeln aktiviert und auf der konvexen Seite die Muskeln entspannt und dehnt. Im Gegensatz dazu wird durch den anstehenden Zügel das Pferd passiv in die Stellung/Biegung geführt, sodass auf der konkaven Seite keine Muskelaktivität stattfindet, somit auch die Muskeln der konvexen Seite keinen (rückenmarksgesteuerten) Impuls zum Loslassen bekommen. In vielen Fällen ist sogar im Gegenteil an der konvexen Seite eine Zunahme der Muskelaktivität sicht- und spürbar in der gleichen Kraftintensität, die der Reiter aufbringt, um die Stellung/Biegung herbeizuführen. Dies tritt insbesondere dann auf, wenn der Körper des Pferdes aufgrund von Verspannungen oder Blockierungen zu einer natürlichen Stellung/Biegung nicht in der Lage ist und diese Bewegung rückenmarksgesteuert ausschaltet, um etwaig auftretenden weiteren Strukturschaden zu vermeiden. Gleiches ist sicht- und spürbar bei Dehnungshaltungen, die das Pferd nicht selbstständig durchführt, sondern die durch die Hand des Reiters forciert werden.

Somit haben wir bei einer nicht korrekt ausgeführten Stellung/Biegung/Dehnungshaltung nicht nur die Situation, dass es nicht den erwünschten Trainingseffekt 


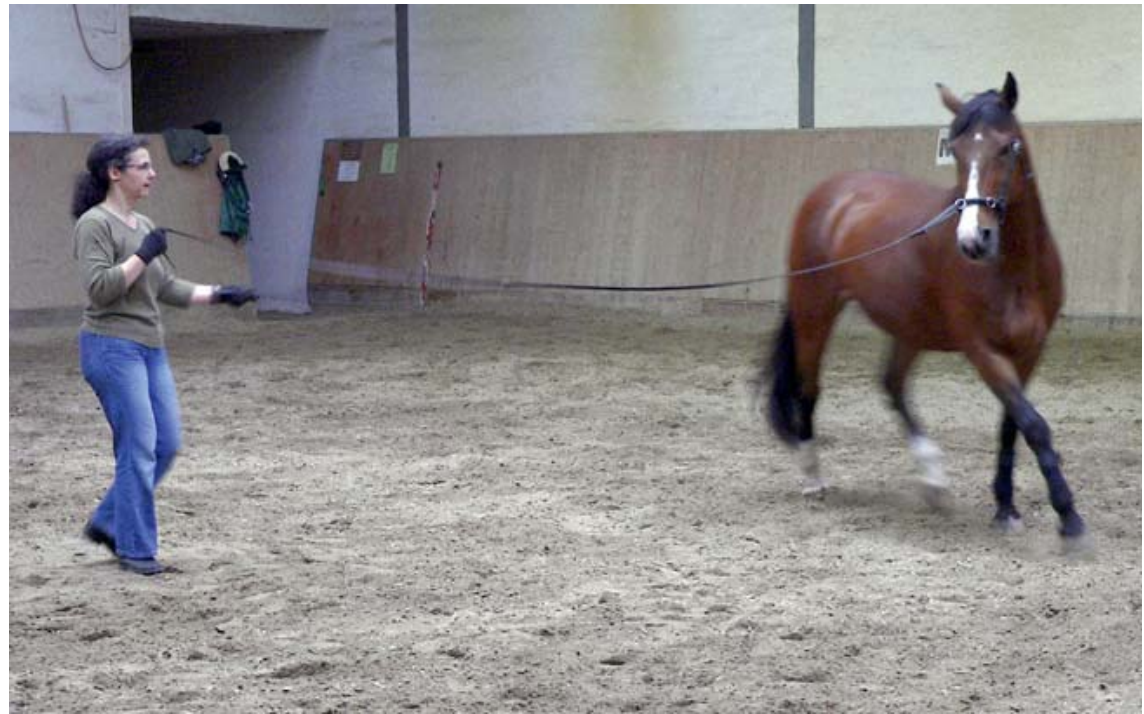

Abb. 2 Beüben der horizontalen Balance von kranial und kaudal ohne Reitergewicht. @ Katja Eser.

im Sinne von Balance, Geschmeidigkeit und Tragkraft bringt, sondern sogar im Gegenteil die unerwünschten Muskelgruppen aufbaut.

Dieses Phänomen von Druck und Gegendruck können wir gut nachvollziehen bei einem kleinen Experiment: Wir stellen uns in aufrechter Körperhaltung mit unserem Gewicht gleichmäßig auf beide Fußsohlen verteilt vor eine Wand und nehmen wahr, an welchen Stellen und mit wieviel Gewicht unsere Füße belastet sind und wie wir in unserem Lot in Balance stehen. Nun heben wir einen Arm an und drücken mit dem kleinen Finger mit wenig Druck gegen die Wand. Wir werden deutlich spüren, wie sich das Gewicht auf unseren Fußsohlen vergrößert und anders verteilt und wie sich die Muskelspannungen in Bauch und Rücken verändern. Selbst der kleinste Druck des kleinen Fingers gegen die Wand zieht einen Gegendruck der Füße in den Boden nach sich.

Auf das Pferd übertragen: Je mehr sich ein Pferd „am Zügel abstößt“, desto mehr schiebt es seine Hinterhand in den Boden hinein und nach kaudal weg - genau das Gegenteil von Hankenbeugung und aktive Lastaufnahme durch die Hinterhand, die wir für einen gesunden Muskelaufbau brauchen.

\section{Praxis}

Die reelle Dehnungshaltung ist das Ergebnis beider gelöster und koordinierter Körperseiten. Die vertikale Balance ist das Ergebnis der Erarbeitung der horizontalen
Balance. Solange eine Körperseite noch steifer bzw. hohler ist als die andere Seite, ist es dem Pferd nicht möglich, eine korrekte, symmetrische, ausbalancierte Dehnungshaltung einzunehmen und zu halten.

Prinzipiell ist es sinnvoll, alle Übungen zunächst in Hand- und Bodenarbeit zu erarbeiten, damit das Pferd die Möglichkeit hat, sich zunächst alleine, ohne „Last“ auf dem Rücken auszubalancieren.

\section{Horizontale Balance von kranial}

Die Stellung im Genick am lösenden Zügel läuft entsprechend der oben beschriebenen Biomechanik durch die Wirbelsäule nach kaudal und führt aufgrund der damit verbundenen LATFLEX zu einem Vorbringen der gleichseitigen Beckenseite.

\section{Horizontale Balance von kaudal}

Das Untertreten eines Hinterbeines unter den Schwerpunkt ist gekoppelt mit einer entsprechenden LATFLEX und gegensinnigen ROT der gleichseitigen Beckenseite, die entsprechend der oben beschriebenen Biomechanik durch die Wirbelsäule nach kranial läuft und im Genick eine gleichseitige Stellung hervorruft.

Das Untertreten der einzelnen Hinterbeine wird erarbeitet über die Seitengänge. Charakteristisch für korrekte Seitengänge ist die Stellung und Biegung des Körpers mit dem Vorbringen der gleichseitigen Hüfte und dem Untertreten eines Hinterbeins unter den Schwerpunkt. Um tatsächlich unter den Schwerpunkt zu tre-
Häufige Sichtbefunde eines unphysiologisch gymnastizierten Pferdes

- Hypertrophie der Kaumuskulatur

- Genickbeule (Hypertrophie der kurzen Genickstrecker bzw. Schwellung der Bursae)

- Hypertrophie der Unterhalsmuskulatur bei Atrophie der Oberhalsmuskulatur

- „Axthieb“ im zervikothorakalen Übergang

- Atrophie von Rücken-, Kruppen- und Oberschenkelmuskulatur

- Senk- oder Karpfenrücken

- kranial ausgestellte oder untergeschobene Vorhand, evtl. mit leicht gebeugtem Karpus

- kaudal ausgestellte oder weit untergeschobene Hinterhand, evtl. mit dauernd wechselnder Entlastung eines Beines

- nach palmar/plantar gebrochene HufFesselbein-Achse, zu lange Zehen, zu niedrige Trachten, evtl. gekoppelt mit unproportional steilerer SchulterblattAchse

ten, ist die Vorwärtskomponente des seitwärts tretenden Hinterbeins unbedingt zu betonen. Ein Kreuzen eines Hinterbeins vor dem anderen führt zu einem Treten vom Schwerpunkt weg, unphysiologischen Scherkräften in den Gelenken der Hinterhand und zu keinerlei Mobilisierung der Wirbelsäule.

Seitengänge werden durchgeführt als Versalverschiebung (Schulterherein, Konterschulterherein) und als Traversalverschiebung (Kruppeherein, Travers, Renvers). Hierdurch ergeben sich für das Pferd „4 Hinterbeine“:

- ein inneres linkes und ein inneres rechtes Hinterbein (Training durch Versalverschiebung)

- ein äußeres linkes und ein äußeres rechtes Hinterbein (Training durch Traversalverschiebung)

Durch das Beüben der „4 Hinterbeine“ des Pferdes können sich eine gleichmäßig starke Tragkraft der Hinterhand, eine symmetrische Dehnungshaltung mit Aktivierung des Nacken-Rückenbands und eine geschmeidige und kräftige Oberlinienmuskulatur entwickeln. Dies befähigt letztendlich das Pferd, unter den gemeinsamen Schwerpunkt mit dem Reiter zu treten 
und diesen auf seinem Rücken zu tragen, ohne reiterlich bedingte gesundheitliche Probleme zu entwickeln.

Sowohl die kraniale als auch die kaudale Erarbeitung der horizontalen Balance an der Hand (\$ Abb. 2) führen zu einer Stellung und Biegung des Pferdekörpers, die später unter dem Sattel dem „Sich-umden-Schenkel-herum-biegen“ des Pferdes entspricht.

\section{Relevanz für Tierärzte und Therapeuten}

Meine Darstellungen über die Biomechanik und deren Konsequenzen für das Training des Pferdes wollen eine Anreiz geben, den Blick zu heben von dem einzelnen erkrankten Gelenk hin zum Betrachten des Pferdes in seinen gesamten Lebens-, Haltungs- und Trainingsumständen.

Eine nachhaltige Besserung oder gar Heilung von Problemen des Bewegungsapparats ist nur dann zu erzielen, wenn neben der akuten Behandlung der Schmerzen, Verspannungen und Blockierungen durch Schul- und Alternativmedizin, Hufschmied, Sattler insbesondere die bisherigen Trainingsmethoden für das Pferd in Augenschein genommen werden. Häufig treten diese alleine schon durch den Sichtund Tastbefund und die Ganganalyse ohne Reiter zutage. Bei chronischen oder rezidivierenden Erkrankungen sollte sich die Zeit genommen werden, dem Reiter bei dem Training mit seinem Pferd zuzuschauen und ihm hilfreiche Hinweise zu geben, an welchen Punkten er seine Arbeit mit dem Pferd verändern muss, um seinen unerlässlichen Teil zur Behandlung seines Pferdes beizutragen.

Die physiologische Gymnastizierung ist gleichermaßen wichtig für Prophylaxe und Genesung.

\section{๑) Summary}

Biomechanics of vertebral column as dictum for horse training: Horses are not necessarily born to be riding horses A horse which exhibits problems such as back pain, lameness, behavioural disorders or problems with organ function should first be examined by an equine veterinarian. However, to identify the real causes of these problems and to bring the horse to a physical, energetic and psychological balance, an osteopathic and energetic examination should also be carried out. In my experience, most problems of the equine musculoskeletal system are due to the unphysiological training of the horse. Long-term continuation of unphysiological training risks the cause of recurring or further symptoms which may result in preventable early death of the horse. Following rigorous manual treatment, further training based on principles of biomechanical and physiological movement is necessary. This is initially carried out by the therapist but can be continued by the rider after learning the principles of physiological training.

Key words

osteopathy - horse - physiological training - back pain - lameness

\section{Literatur}

[1] Eser K. Checkliste Osteopathie des Pferdes. Stuttgart: Sonntag Verlag in MVS Medizinverlage Stuttgart; 2011

Online zu finden unter

http://dx.doi.org/10.1055/s-0031-1298427

() Katja M.-L. Eser

Human-Physiotherapeutin, Pferde-Osteopathin Judmannstraße 1

85049 Ingolstadt

E-Mail: Katja.Eser@horsehealing.de

Jahrgang 1967, 1994 Amtsarztprüfung Humanheilpraktikerin, 2002 Staatsexamen Humanphysiotherapie, 2004 Tierheilpraktikerin (ATN), 2006 Pferdeosteopathie (EPOS), 2009 Equine Lymphdrainage nach Prof. Rautenfeld (TiHo Hann.). Seit 1994 in selbstständiger Naturheilpraxis, seit 2002 zusätzlich freiberuflich in Physiotherapiepraxis tätig. Seit 2006 selbstständige Fahrpraxis mit Schwerpunkt Osteopathie und Gymnastizierung für Pferd und Reiter. 2007-2010 Dozentin für Pferdetherapeuten in Ingolstadt. 2011 Veröffentlichung des Buches „Checkliste Osteopathie Pferd“ im Sonntag Verlag. 